\title{
EDITORIAL
}

\section{Stroke alert: sleep disordered breathing predicts survival?}

\author{
L. Grote
}

Stoke is a very widespread disease, a leading cause of death, and generates high healthcare costs due to causing considerable disability. The aetiology and outcomes of stroke are various. Ischemic stroke dominates and differs pathogenetically from haemorrhagic stroke, the latter having a poorer prognosis. Several modifiable risk factors, including hypertension, diabetes, hyperlipidemia, smoking, and arterial fibrillation, have been defined in stroke. However, these traditional risk factors do not fully explain the occurrence of stroke and new risk factors including inflammatory markers, infection and homocysteine have been proposed [1]. Other data have identified sleep disordered breathing (SDB) as a potential risk factor.

SDB is a common condition affecting $10-20 \%$ of the adult population and an even higher incidence has been reported in the elderly. Obstructive sleep apnoea (OSA) is by far the most common type of SDB, whereas central SDB, such as CheyneStokes respiration, is more frequent in patients suffering from acute stroke and cardiac failure. There is ongoing debate on the causal role of SDB in vascular disease. Several epidemiological studies identified an association between SDB and ischemic heart disease and stroke, but the causality remained unresolved. Recent data on the incidence of fatal and nonfatal cardiovascular events clearly suggest that coexisting SDB increased event rate [2,3]. The risk impact varies between studies but is generally low-to-moderate. However, the high prevalence of SDB implied that even a minor risk increase may have considerable implications for public health. The epidemiological data contrasts with results from pathophysiological case control studies mainly performed in OSA patients. These studies suggest a far stronger link between SDB and vascular disease. OSA has a profound cardiovascular impact by increased sympathetic activity, reduced vascular endothelial function, neuro-endocrine activation, and as a trigger of systemic vascular inflammation. The resulting haemodynamic instability and recurrent nocturnal oxygenation/re-oxygenation have been proposed as the main trigger mechanisms for vascular and cardiac stress during sleep. Other studies have demonstrated that elimination of OSA by nasal continuous positive airway pressure (CPAP) normalised vascular or cardiac dysfunction, hypercoaguable state, oxygen radical release and inflammatory cell activation. However, it should be kept in mind that far from all SDB/ OSA patients appear to develop a manifest cardiovascular disease even after years of apnoeic events. It is therefore likely that yet unidentified compensatory mechanisms of geneticl environmental origin play an important role for susceptibility of vascular disease in SDB patients.

Patients with manifest cerebrovascular disease (ischaemic or haemorrhagic lesions) constitute a high-risk group prone to excess mortality. The role of SDB in this group has been extensively investigated [4]. Early pioneer work found an

Correspondence: L. Grote, Sleep Disorders Centre, Dept of Pulmonary Medicine, Sahlgrenska University Hospital, Gothenburg, Sweden. Fax: 46 31824904. E-mail: ludger.grote@lungall.gu.se over-representation of snoring and or diagnosed SDB in patients after stroke. Subsequent work suggested that up to $50 \%$ of patients with manifest stroke, but not with transitory ischaemic attacks (TIA), have SDB, thereby indirectly indicating a potential influence of SDB in stroke aetiology. A recent large-scale epidemiological study, the Sleep Heart Health Study, reported a linear association between stroke and SDB, even steeper than that found for cardiac disease [5]. Moreover, this study could not determine whether OSA/SDB preceded the event or if it occurred as a result of the stroke disease. Incident data on stroke in SDB patients are expected to be reported soon from this study and may shed light on the causal relationship of the association. One dominant risk factor for cerebrovascular disease in this context may certainly be OSA-induced systemic hypertension. This form of hypertension has been well-documented in several epidemiological and controlled clinical studies. However, beside this and other adverse vascular impact mentioned above, SDB may increase stroke risk and worsen recovery after stroke by factors, such as decreased cerebral blood flow during OSA events, altered cerebrovascular autoregulation, and increased susceptibility for paradoxical embolisation via an open foramen ovale during obstructed breathing. In addition, two treatment studies of SDB in poststroke patients showed improved rehabilitation outcome and mood stability $[6,7]$.

In the current issue of the Journal, PARRA et al. [8] reported follow-up data on 161 patients with first-ever stroke $(\mathrm{n}=122)$ or TIA $(n=39)$ after an observational period of $\sim 2$ yrs. In total, 22 patients died during this period and vascular disease $(63 \%)$, in particular the reoccurrence of a cerebrovascular event $(50 \%)$, was the dominant cause of death. Traditional risk factors such as age, diabetes, ischaemic heart disease and cardiac failure, as well as pulmonary disease, came out as predictors for mortality in the univariate statistical model. Most interestingly, the number of SDB events, obtained from a limited sleep study during the acute phase of the stroke/TIA event, was a powerful predictor for $2-y r$ mortality. In the multivariate model, only SDB (apnoea-hypopnoea index, 5\% risk increase per unit), age, infarct localisation with involvement of the middle cerebral artery, and concomitant ischaemic heart disease came out as independent predictors. The occurrence of predominantly central SDB was associated with particular mortality. This is the first prospective study addressing the role of SDB in stroke mortality. Its strengths include the large number of patients and the assessment of SDB during the acute phase of stroke. In addition, the study provides a long-term follow-up, an exact identification of different potential confounders (traditional risk factors, type and localisation of vascular damage, physical and mental health state by questionnaire data etc.) and the classification of different sleep variables. Limitations include the lack of an experimental or interventional study arm. Thus, it would have been of specific interest to document the role of SDB treatment (e.g. nasal CPAP, oral devices, drug treatment) in this patient group in order to further address a causal role of 
SDB in stroke mortality. The follow-up data were not based on regular scheduled follow-up visits. No detailed data were provided on the number of TIA/stroke relapses, the incidence of other cardiovascular disease, drug treatment logs, or on the estimation of compliance with risk-modifying drug treatment. It may be argued that the occurrence of SDB only provides a measure of a generally poorer health status of the patients and not a mechanism for increased risk on its own. However, the current study from PARRA et al. [8] should be seen as an important milestone for the understanding of the potential role of SDB in patients with acute cerebrovascular disease.

What are the important insights to be gained from this study and what are the new scientific questions that have been raised? 1) An important risk predictor for stroke outcome can be identified in a sleep study within $72 \mathrm{~h}$ after the acute stroke/ transitory ischemic attacks event. This finding may have considerable implications for future stroke management if the results can be confirmed in larger trials. Multicentre studies would be a possible scenario, including stroke units capable to implement sleep studies, as a routine procedure in acute stroke care. With even larger patient numbers it may be possible to identify the predictive value of central versus obstructive sleep disordered breathing after stratification for age, different stroke aetiologies and possibly even different stroke localisations. 2) Randomised treatment trials are warranted to verify whether treatment of sleep disordered breathing may reduce stroke mortality. If sleep disordered breathing as a mortality predictor cannot be modified or reversed by treatment, diagnostic evaluation certainly becomes less relevant. Different treatment modalities (nasal ventilation, oxygen therapy, antioxidative treatment, improvement of endothelial function etc.) could be investigated as acute or long-term intervention in stroke patients with sleep disordered breathing. 3) PARRA et al. [8] used a validated multichannel recorder to assess sleep disordered breathing in the acute setting. This diagnostic tool in the hands of trained and experienced personnel may be practical and produce valid results. However, specific development of new, simplified diagnostic tools or the validation of already existing equipment in the specific setting of acute stroke care may be of particular interest to assess adequate markers of sleep disordered breathing in the context of stroke. 4) This study suggests that sleep disordered breathing monitoring in the acute phase of stroke predicts mortality. It would be of interest to compare these findings with data obtained from monitoring performed during a later stage of rehabilitation. Monitoring during the acute phase of stroke may be particularly problematic, at least in some subgroups of patients.

Acknowledgements. The author would like to thank J. Hedner for kind assistance during the writing of this Editorial.

\section{References}

1. Diaz J, Sempere AP. Cerebral ischemia: new risk factors. Cerebrovasc Dis 2004; 17: 143-150.

2. Peker Y, Hedner J, Norum J, Kraiczi H, Carlson J. Increased incidence of cardiovascular disease in middle-aged men with obstructive sleep apnea: a 7-year follow-up. Am J Respir Crit Care Med 2002; 166: 159-165.

3. Lindberg E, Janson C, Svardsudd K, Gislason T, Hetta J, Boman G. Increased mortality among sleepy snorers: a prospective population based study. Thorax 1998; 53: 631637.

4. Hermann DM, Bassetti CL. Sleep-disordered breathing and stroke. Curr Opin Neurol 2003; 16: 87-90.

5. Shahar E, Whitney CW, Redline S, et al. Sleep-disordered breathing and cardiovascular disease: cross-sectional results of the Sleep Heart Health Study. Am J Respir Crit Care Med 2001; 163: 19-25.

6. Wessendorf TE, Wang YM, Thilmann AF, Sorgenfrei U, Konietzko N, Teschler H. Treatment of obstructive sleep apnoea with nasal continuous positive airway pressure in stroke. Eur Respir J 2001; 18: 623-629.

7. Sandberg O, Franklin KA, Bucht G, Eriksson S, Gustafson Y. Nasal continuous positive airway pressure in stroke patients with sleep apnoea: a randomized treatment study. Eur Respir J 2001; 18: 630-634.

8. Parra O, Arboix A, Montserrat JM, Quinto L, Bechich S, Garcia-Eroles L. Sleep-related breathing disorders: impact on mortality of cerebrovascular disease. Eur Respir J 2004; 24: $267-272$. 